\title{
Observations of coronal streamers on the night sky
}

\author{
R.A. Gulyaev ${ }^{1}$, D. Kokotanekov ${ }^{2}$ and N. Petrov ${ }^{2}$ \\ ${ }^{1}$ IZMIRAN, Troitsk, Moscow Region, Russia; email: rgulyaev@izmiran.troitsk.ru \\ ${ }^{2}$ Institute of Astronomy, Bulgarian Academy of Sciences, Sofia, Bulgaria
}

Abstract. We report on detection of coronal streamer trace on the night sky over the horizon.

50 years ago, Nikolsky (1956) had paid attention to feasibility of observations of solar coronal streamers on the night sky. According to his estimate, a coronal streamer may be as bright as the zodiacal light at the elongation of $50^{\circ}$ from the Sun. In 1956-1957 he had tried to detect coronal streamers on the night sky using the Schmidt camera but endeavours met in failure.

Gulyaev had now and again searched for coronal streamers on the night sky using powerful lenses and image amplifiers. At night on 6 to 7 June 1994 an extended faint luminous feature has been detected in the Camelopardalis constellation. The formation was oriented approximately perpendicular to the direction towards the Sun. The observed phenomenon was expected to be the trace of a coronal mass ejection (Gulyaev 1996).

Finally, we have apparently first succeeded in recording the trace of a coronal streamer on the night sky over the horizon in October 2002. Observations have been carried out at Rozhen Observatory of Bulgarian Academy of Sciences. We have the main task of photographic and interferometric (in the $K$ Ca II line) observations of the morning zodiacal light. Fortunately, some photographs show a faint luminous feature in addition to the zodiacal light image.

A suitable photograph was obtained on 9 October at $3 \mathrm{~h} 02 \mathrm{~m} \mathrm{UT,} 80 \mathrm{~min}$ prior to sunrise. We used a lens of $F / 2.8, F=20 \mathrm{~mm}$ and the Kodak TMax-3200 film; the exposure time was $20 \mathrm{~s}$. The dip of the Sun below the horizon has been about $17^{\circ}$. The negative shows the zodiacal light in the Leo constellation. North, a faint luminous band is adjoined to the zodiacal light cone. Extrapolation of the band under the horizon fits well a streamer at position angle of about $-72^{\circ}$. (Location of streamers around the Sun is known from the coronal image obtained at the same time with the LASCO-C3 coronagraph at the SOHO space observatory). The fact of such a coincidence gives ground to interpret the luminous band as an extension of the coronal streamer.

The photograph was subjected to computerized reduction in order to built up a map of isophotes. One typical isophote is shown in figure 1. It is clearly seen a bulge produced by superposition of extended coronal streamer and zodiacal light. In figure 1 there are indicated also directions of horizon and ecliptic and location of the Sun.

Our negatives have not photometric calibration, but we can estimate intensities referring to the standard model of brightness distribution in the zodiacal light. We used the modern data by Levasseur-Regourd et al. (2001). The most prominent portion of the extended streamer at angular distance of $46^{\circ}$ from the Sun has been analysed. The proper brightness of the streamer in this point was found to be equal to $200 S_{10}$ or $0.85 \times 10^{-13}$ of the mean brightness of the Sun.

It should be noted the following. It is known nowadays that large coronal streamers represent structural elements of the heliospheric current sheet. So, observations of coronal 


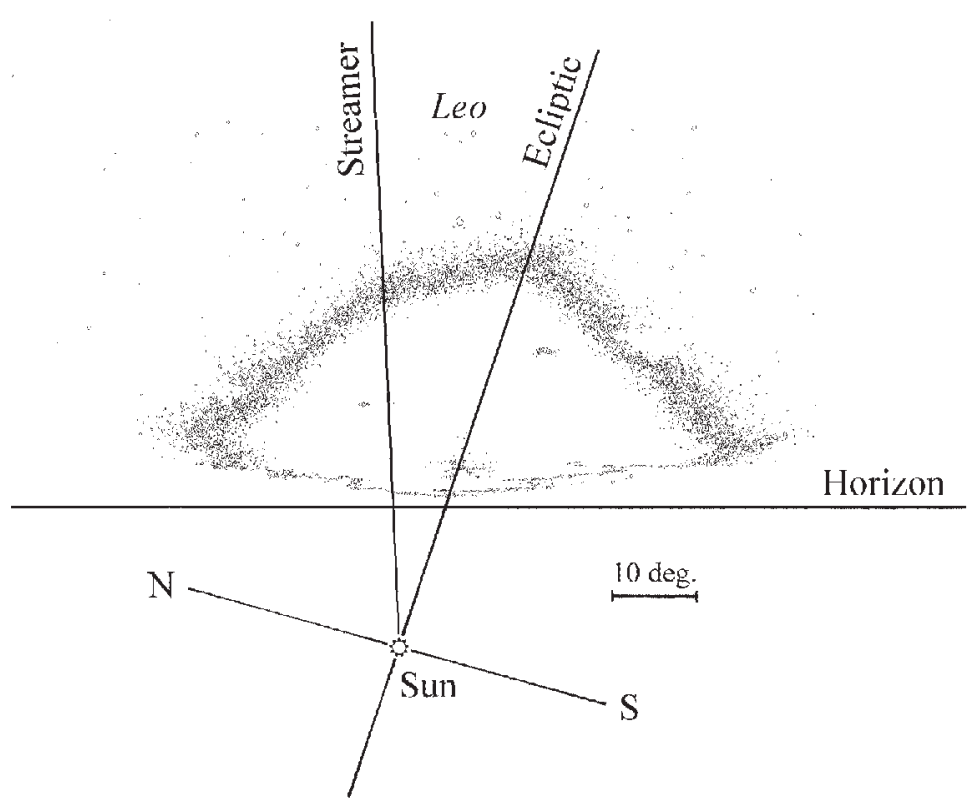

Figure 1. One of isophotes of the total emission of the zodiacal light and the streamer extension. The situation on celestial sphere is shown too. NS is projection of the Sun's rotation axis. Out of the isophote one can see representation of a number of stars. A bulge at the left portion of the isophote refers to the streamer extension.

streamers on the night sky would provide for the direct optical recording of the HCS in large heliocentric distances and at wide range of heliographic latitudes. Ground-based optical monitoring of the HCS might be planned in prospect.

At present, the task of search for coronal streamers on the night sky must be more readily since permanent monitoring of the outer solar corona at space stations (first of all, at the SOHO observatory) allows obtaining suitable information on the streamer locations on the celestial sphere under real-time conditions.

\section{Acknowledgements}

This work is partially supported by the National Scientific Found (Bulgaria) under Grant 1004/00.

\section{References}

Gulyaev, R.A. 1996 Adv. Space Res. 17, No.4/5, 315-318.

Levasseur-Regourd, A.C. et al. 2001 In Interplanetary Dust (ed. E.Grun, B.A.S.Gustafson, S.Dermott, \& H.Fecting), pp. 57-94. Springer.

Nikolsky, G.M. 1956 Astron. Zh. (Russian) 33, 588-598. 